\title{
Neurodegeneration halted with oral therapy in mice
}

An oral therapy that targets the unfolded protein response (UPR) has been shown to prevent neurodegeneration in a mouse model of prion disease. The treatment abrogated the behavioural, clinical and pathological characteristics associated with accumulation of misfolded prion protein. Notably, Alzheimer disease, amyotrophic lateral sclerosis and Parkinson disease are also associated with a build-up of misfolded proteins and activation of the UPR. "We may have found a common pathway to target all of these disorders," says Giovanna Mallucci, lead author of the new study.

Previous research by the team showed that accumulation of misfolded prion protein in the mouse brain overactivates the UPR and results in the sustained shut down of protein synthesis, a decline in key synaptic proteins and, ultimately, neurodegeneration. The key player in the pathway is protein kinase RNA-like ER kinase (PERK). PERK phosphorylates the eukaryotic initiation factor 2 (eIF2) a subunit, which prevents the initiation of translation. Genetic inhibition of this pathway protected against neurodegeneration in prion diseased mice. In this study, Mallucci and colleagues assess the effects of GSK2606414, a pharmacological inhibitor of PERK.

$\mathbf{4}$ We may have found a common pathway to target all of these disorders... 77

Mice were inoculated with prions at 4 weeks of age. They were then treated with GSK2606414 at either 7 weeks after inoculation $(n=20)$, when prion infection is established in the brain but before memory and behavioural signs occur, or 9 weeks after inoculation $(n=9)$, when behavioural signs and prion pathology are present. All control animals $(n=17)$ were terminally ill 12 weeks after inoculation and had clinical signs of prion disease, whereas treated animals were free from diagnostic clinical signs.

Histopathological analysis revealed evidence of neuroprotection in the brains of the treated mice compared with controls, which had extensive neuronal loss and spongiform degeneration. Levels of both phosphorylated PERK and eIF2 $\alpha$ were also reduced, and vital synaptic protein levels were maintained at normal levels, in the animals that received GSK2606414. Adverse systemic effects of the compound included weight loss and elevated blood glucose.

These data support PERK inhibition as a target for preventing the reduction of protein synthesis that leads to neurodegeneration. "What is very exciting is finding a potentially generic pathway, the inhibition of which is so effective in preventing neurodegeneration," says Mallucci. Much more work will be needed, however, before the implications of this research are realized in the clinic. The next steps will be to confirm the pathway in patients, and to refine drug development.

Bryony Jones

Original article Moreno, J. A. et al. Oral treatment targeting
the unfolded protein response prevents neurodegeneration
and clinical disease in prion-infected mice. Sci. Transl. Med.
doi:10.1126/scitranslmed.3006767

